\title{
Ein Problem der Dynamik
}

\author{
Von
}

\section{J. Tinbergen, Scheveningen}

\section{Einführung}

1. Die für das Verständnis der reellen wirtschaftlichen Bewegungen notwendige Erweiterung der statischen Theorie der Sozialökonomie wird von einer wachsenden Zahl von Forschern unternommen, und bedeutende Resultate sind in dieser Hinsicht sschon erreicht worden. Als einige der wichtigeren Stufen in dieser Entwicklung seien genannt:

a) Die Erweiterung der statischen Theorie zur komparativ statischen. Ausgehend von den mathematischen Formulierungen der fast allgemein anerkannten Gleichgewichtstheorie kann man diese komparativ statische so charakterisieren, daß statt bestimmter Konstanten jetzt gegebene Funktionen der Zeit in den Gleichgewichtsgleichungen figurieren. Durch diese in der Zeit veränderlichen Parameter, werden die Gleichgewichtswerte der Preise und Umsätze gleichfalls veränderlich. Auf dieser Konstruktion basieren bekanntlich eine große Zahl von Analysen von Nachfragen- und Angebotskurven ${ }^{\mathbf{1}}$ ).

b) Die Erweiterung des komparativ statischen Bildes durch Heranziehung der Verzögerungen, der "Lags", der „Zeitqualität" (Morgenstern). Es ist speziell die Konjunkturforschung, welche sich mit Erfolg dieser Konstruktion bedient hat. Das interessante an dieser Theorie besteht speziell darin, daß in ihr endogene Bewegungen möglich werden,

1) Ohne Anspruch auf Vollständigkeit seien die folgenden Arbeiten aus letzter Zeit genannt: Donner: Bestimmungsgrŭnde der Baumwollpreise, Vierteljahrshefte zur Konjunkturforsehung, Sonderheft 15; Ezekiel: Preisvoraussage bei landwirtschaftlichen Erzeugnissen (wo auch seine amerikanische Arbeiten zu finden sind), Veröffentlichungen der Frankfurter Gesellschaft für Konjunkturforsehung, Heft 9 (1930); Leontieff: Ein Versuch zur statistischen Analyse von Angebot und Nachfrage, Weltw. Archiv, Bd. 30, 1929; Moore: Synthetic Economics (worin weitere Zitate); De Nederlandsche Conjunotuur („Die Niederländische Konjunktur", herausgegeben vom holländischen statistischen Zentralamt), 1930/31; Warren and Pearson: Interrelationships of Supply and Price, Bulletin 466, Cornell University Agricultural Experiment Station, 1928; Pigou: The Statistical Derivation of Demand Curves, Economic Journal, 1930, p. 384; Schultz: Statistical Laws of Demand and Supply, Chicago, 1929; Staehle: Die Analyse von Nachfragekurven in ihrer Bedeutung für die Konjunkturforschung, Veröffentlichungen der Frankfurter Gesellschaft für Konjunkturforschung, Heft 2, 1929.

Zeltschr. t. Nationalókonomie, IIr. Bd., 2. H. 
d. h., daß ohne die Bewegung äußerer Größen Bewegungserscheinungen möglich werden ${ }^{1}$ ).

c) Die Einführung von Preisableitungen in den Nachfrage- oder Angebotsfunktionen. Hiedurch hat man angeben wollen, da $B$ in der dynamischen Wirklichkeit neben dem Preisstand auch der Preisverlauf auf Nachfrage und Angebot einwirken kann ${ }^{2}$ ).

Es sind, soviel ich weiB, hauptsächlich diese drei mathematischen Figuren, welche in der statistisch-ökonomischen Forschung angewandt worden sind. Alle Forscher werden wohl darüber einig sein, daB die Vorstellungen, die man über den dynamischen Ablauf wirtschaftlicher Vorgänge hat, schon bedeutend weiter entwickelt sind, als in diesen ziemlich einfachen Konstruktionen der Interdependenz zum Ausdruck kommt; man hat diese weiteren Vorstellungen aber noch kaum zu einer systematischen Gestaltung der dynamischen Theorie zusammengefaßt: zu einem Schema, das sich an das fast allgemein angenommene Schema der statischen Theorie anpaßt, wie es z. B. Bowley in seinem ,Groundwork" gegeben hat. Im folgenden wird versucht, die Elemente und einige Überlegungen zu einem solchen Schema zu geben, wobei aber nicht mehr als eine rohe Skizzierung möglich sein wird (Abschnitt II). Daneben wird die Lösung eines äußerst einfachen konkreten Problems mit dergleichen Utberlegungen versucht (Abschnitt III).

\section{Das dynamische Marktschema}

2. Aufgabe eines Marktschemas ist die Lösung folgenden Problems:

Gegeben sind eine Zahl von wirtschaftlichen Subjekten (Personen, Unternehmen), welche den betreffenden Markt bilden, und die Werturteile dieser Tauschenden. Gegeben sind weiter bestimmte natürliche und technische Bedingungen (z. B. Produktionskoeffizienten) und wirtschaftliche Bedingungen (die Gleichgewichtsbedingung: ,Nachfrage = Angebot", und die Form der "Marktorganisation": ob Monopol oder Konkurrenz herrsche usw.), welchen die gesuchten Größen genügen sollen. Die Werturteile der Subjekte sind gegeben in Form der sogenannten Ophelimitätsfunktion, worin als Veränderliche die Güterströme vorkommen, welche in einer Zeiteinheit dem Subjekt zuflieBen oder vom Subjekt abfließen ${ }^{3}$ ).

Gesucht sind die von den einzelnen Subjekten getauschten Mengen und der Preis, zu welchem der Tausch vor sich gegangen ist.

1) Vor allem sei hier hingewiesen auf Hanaus, ,Prognose der Schweinepreise" (Sunderheft 18 der Vierteljahrshefte zur Konjunkturforschung) und auf die theoretische Behandlung in Moores "Synthetic Economics". Vgl. auch meine Arbeit im Weltw. Archiv, 1931: "Ein Schiffbauzyklus ?"

2) Amoroso: Intorno alla determinazione empirica delle leggi della domanda e dell'offerta, Giornale degli Economisti, November 1930, S. 941, und frühere Arbeiten; Roos: A Mathematical Theory of Competition, Am. Journal of Math., XLVII (1925), S. 163; Evans: A Simple Theory of Competition, Am. Math. Monthly, XXIX (1922).

3) Die ökonomisch vielleicht wichtigste Spezialform der Ophelimitätsfunktion ist die, welche den Gewinn eines Unternehmers darstellt. 
Für die Art wie beim statischen Schema die Lösung gefunden wird, kann auf zahlreiche Lehrbücher verwiesen werden, vor allem auf Bowleys "Groundwork"s. Wir wollen aber jetzt skizzieren, wie dieses Schema zu einem dynamischen erweitert werden kann. Dazu bedarf es einiger neuen Begriffe, die wir zuerst kurz einführen wollen.

3. Das Gesichtsfeld. Wenn nicht mehr, wie in der Statik, das Konstantbleiben der ökonomischen Größen vorausgesetzt wird, wird auch der Umstand beeinflußt, daß jedes Subjekt bei seinen Dispositionen nur mit der nächsten Zukunft rechnet und daher, was dem Zeitablauf anbetrifft, sei es bewußt oder unbewußt, ein beschränktes "Gesichtsfeld" hat. Im allgemeinen wird sein Interesse für spätere Zeitpunkte und die diese beeinflußenden Größen je nach ihrem Abstand schwächer werden: es ist die von Böhm-Bawerk eingeführte ,perspektivische Verkleinerung". Obgleich also von einem genau begrenzten Gesichtsfeld im allgemeinen nicht gesprochen werden kann, so hat es doch zur Vereinfachung in gewissen Fällen, wie es mir scheint, wohl einen Sinn, von einer derartigen Fiktion auszugehen und dem Gesichtsfeld eine genaue Dauer zuzuschreiben ${ }^{1}$ ). (Vgl. dazu: das Beispiel im III. Abschnitt.) Es scheint dann weiter, daß diese Dauer für dieselbe Person für verschiedene Teilgebiete seiner wirtschaftlichen Tätigkeit ganz verschieden sein kann. Man pflegt beim Beurteilen des Baues einer Fabrik viel weiter in die Zukunft zu schauen, als bei der Einkaufspolitik der Rohstoffe usw. Die Ursachen dieses Unterschiedes sind $\mathrm{m}$. $\mathbf{E}$. in der sehr verschiedenen Lebensdauer verschiedener ,Güter" (im weitesten Sinne) zu suchen und dazu in der versehiedenen Unsicherheit der Voraussage einzelner Wirtschaftsabläufe. Als konkrete Beispiele für diese Gedanken seien schließlich einige Zahlen in Erinnerung gebracht, welche mit dem Umfang des Gesichtsfeldes zusammenhängen dürften. Die Rohstoffbestände in den meisten Fabriken kommen im allgemeinen dem Verbrauch für einige Monate gleich: die Baumwollbestände bei den Spinnereien z. B. sind ungefähr gleich dem Verbrauch von 2 bis 3 Monaten. Termingeschäfte werden maximal auf 9 bis 12 Monate abgeschlossen. Demgegenüber steht die so viel längere Lebensdauer von allerhand Produktionsmitteln, die sich bis auf mehrere Jahrzehnte oder bei Bauten sogar auf Jahrhunderte erstreckt.

4. Die „Erwartung“. Während im statischen Schema immer nur Größen vorkommen, deren numerischer Wert bei allen Subjekten als bekannt vorausgesetzt wird - alle Größen, die in der Zukunft bestehen werden, haben da eben denselben Wert wie in der Vergangenheit -, kommt in der Dynamik prinzipiell der Umstand dazu, daß bestimmte zukünftige Größen nicht bekannt sind und die Subjekte deshalb nur mit der Erwartung, die sie bezüglich des Umfangs dieser Größen haben, rechnen können. So z. B. wird das Angebot eines dynamisch reagierenden Subjektes nicht nur durch die Preise, sondern auch durch die Preiserwartungen für spätere Zeitabschnitte (in die wir uns das Gesichtsfeld

1) Wir wollen diese Dauer im folgenden mit $\tau$ angeben. 
unterteilt denken) beeinflußt. Neben Preiserwartungen können auch Erwartungen über andere Größen, z. B. Ernten, in der Ophelimitätsfunktion vorkommen. Es scheint $\mathrm{mir}$, daß die besondere Bedeutung, die diese Erwartungen für die theoretische Ökonomie haben, eine spezielle Schreibweise dieser Größen rechtfertigen würde; ich möchte daher vorschlagen, da. $\mathbf{B}$ sie $\mathbf{z}$. B. durch gotische Buchstaben angegeben werden mögen. Im folgenden wird diese Schreibweise versuchsweise angewandt werden.

Die ,Erwartungen" weisen gegenüber den ,wirklichen" Größen einen wichtigen Unterschied auf. Sie können nämlich auch bei vollkommener Information, für einzelne Subjekte verschieden sein; was dann zu sehr komplizierte Möglichkeiten führen kann. Inzwischen werden wir bei unserem Beispiel im III. Abschnitt annehmen, daB die Erwartungen bezüglich einer bestimmten Größe für alle Subjekte die gleichen sind. Die nach meiner Ansicht wesentlichste Eigenschaft der „Erwartungen" ist damit keineswegs eliminiert: diese besteht m. E. vielmehr darin, daß sie nicht Wirklichkeit zu werden brauchen, weil neue Tatsachen, die bis dahin unbekannt waren und es auch sein muBten, sie beeinflussen. Wir werden sogar noch einen Schritt weiter gehen und auch annehmen, daß die Erwartungen ,,vernünftig" sind, d. h. mit den wirtschaftlichen Zusammenhängen übereinstimmen. Was die genauen Folgen dieser Annahme bedeuten, ist am besten an einem Beispiel zu zeigen. Die letzte Annahme widerspricht übrigens nicht der soeben angegebenen essenziellen Eigenschaft der Erwartungen.

In bestimmten Fällen - und das werden vorläufig wohl die meist erfolgreichen bei der Analyse sein - kann man die "Erwartungen" durch wirtschaftstheoretische Deduktion, durch bestimmte Konstanten oder reelle Größen ersetzen. Handelt es sich z. B. um eine Erwartung bezüglich einer Größe, welche eine ,zufällige Variable“ ist, so ist die vernünftige Erwartung die mathematische und also eine bestimmte Konstante. Ein anderes Beispiel bildet die Erwartung einer Größe, welche mit einer gewissen Annäherung, einem bestimmten Gesetz gehorcht. Die Erwartung kann dann durch eine Reihe, in der die Gegenwartswerte dieser Größe und ihrer Ableitungen nach der Zeit vorkommen, ersetzt werden. So kann man auch die unter Io genannten Nachfragen- und Angebotsfunktionen - als erste Näherungen - begründen. Schließlich sind dann zwischen diesen beiden extremen Beispielen allerhand Zwischenfälle denkbar.

Wir wollen unsere Bemerkungen über die Erwartungen nicht abschließen, ohne noch eine kleine statistische Illustration einiger Erwartungen zu geben. Man kann nämlich mit einem gewissen Recht die Terminnotierungen als solche betrachten. In Abbildung 1 habe ich zwei Reihen von Terminnotierungen graphisch dargestellt. Als Erklärung möge auf die Unterschrift der Abbildung verwiesen werden. Das uns wichtigste an der Figur ist wohl, daB im allgemeinen die Terminnotierungen mit den wirklichen Preisen im Augenblick, wo sie notiert werden, besser übereinstimmen als mit den Preisen, deren Voraussage sie sozusagen 
sein wollen $\left.{ }^{\mathbf{1}}\right)$. Das heißt: im allgemeinen ist die Preiserwartung gleich dem Augenblickspreis, oder in knappen Jahren natürlich niedriger als dieser; höher aber nur, wenn die Nachfrage für das nächste Jahr höher angenommen werden muß. Im III. Abschnitt hoffe ich darzulegen, daß dieses Verhalten mit der da gegebenen vereinfachten "Abbildung" in ziemlich gutem Einklang steht.

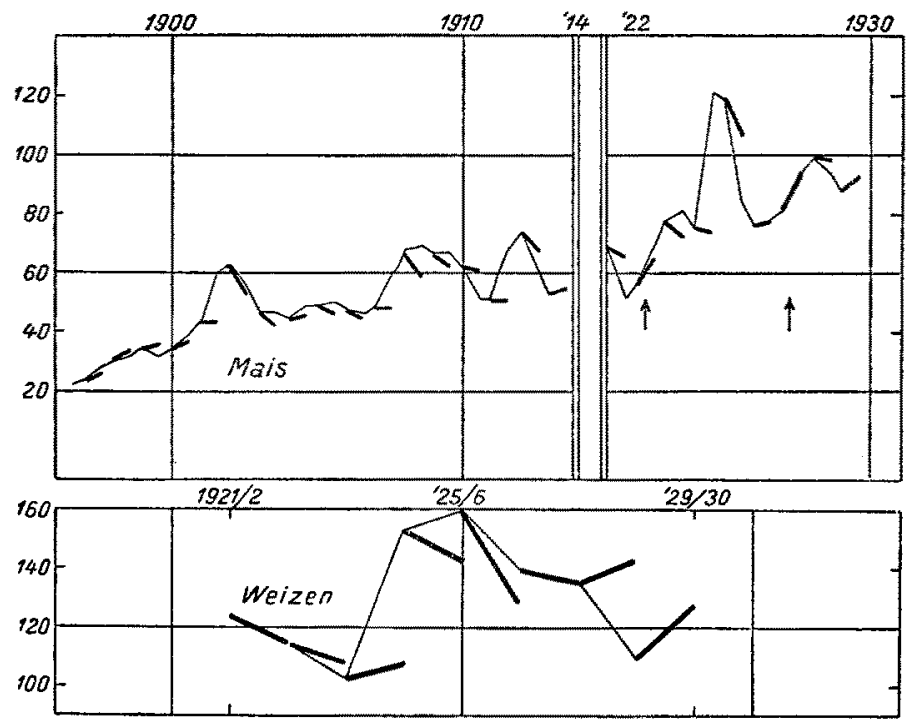

$\mathrm{Abb} .1$

- Wirkliche Preisbewegung (Vereinigte Staaten)

Vorhergesagte Bewegung nach den Terminpreisen

Fur Mais sind eingezeichnet worden: au? den Jahregvertikalen der Durchschnitt des Preises für Dezember des Vorjahres und Mal des betreffenden Jahres; dazwischen die Durchschnitte der Preise für September und Dezember. Die angegebenen Terminnotierungen (die rechten Eudpunkte der dicken Liniensegmente) sind Durchschnitte aus den Terminnotierungen für September $\mathrm{lm}$ Mârz und für Dezember im Mai, d. h. sie stellen ,, Vorheraagen in das neue Erntejahr" dar. Man sleht, in Uberelnstimmung mit den Ausführungen in \& 8, da日 stark aufwäts gerichtete Vorhersagen selten vorkommen; In den zwel mit Pfellen angegebenen Jahren war der Schwejnebestand im schnellen Anwachsen, was als eine ,äußere“ Ursache für die Steigung angegehen werden dürfte. Man sieht gleichfalls, da. die Korrelation zwischen den "vorhergesagten* und den wirklichen Preisen am besten ist, wenn man die vorhergesagten eín Jahr zurückschjebt, also vergleicht mit den augenblicklichen Preisen

Quellen: Mais: Farm Economics; Weizen: Wheat Futures, Dept. of Commerce

5. „Verzögerte" natürliche und technische Bedingungen. Während wieder in der Statik wegen des Konstantbleibens aller Größen eventuelle Verzögerungen auch unsichtbar werden, ist dieses in der Dynamik nicht der Fall und tritt die Bedeutung dieser Erscheinung viel stärker in den Vordergrund. Die besonderen Eigentümlichkeiten und Folgen sind aber zu bekannt, als daß hier noch näher darauf eingegangen werden soll.

1) Auch für Baumwollterminpreise trifft dies in der Hauptsache zu. Siehe Forrest Bee Ashby im Journal of the Am. Stat. Ass. XXIV (1929), S. 412 . 
Wir wollen es bei diesen Andeutungen bezüglich neuerscheinenden Begriffen bewenden lassen und nun noch eine flüchtige Skizze des Marktschemas selbst geben. Wenn die Ophelimitätsfunktionen gegeben eventuell aufgestellt worden - sind, wobei dann also auch das Gesichtsfeld bekannt ist; wenn weiter die Preiserwartungen für alle Zeitabschnitte, welche berücksichtigt werden, gegeben oder abgeleitet worden sind, so bestimmt demnach jedes Subjekt eindeutig sein Angebot bzw. seine Nachfrage für jeden dieser Zeitabschnitte. Es macht sich also einen „Wirtschaftsplan", charakterisiert durch eine Reihe verschiedener Angebots- oder Nachfragewerte. Benennen wir die Zeitabschnitte nach ihren Anfangspunkten und zählen wir die Zeit von dem Augenblicke an, wo unser Problem beginnt, so werden also für die Zeitabschnitte 0 bis $n$-1, wobei $n$ der Umfang des Gesichtsfeldes sein möge, die Angebots- oder Nachfragenmengen geplant, sagen wir auf

$$
x_{00}, x_{01}, x_{02}, x_{03} \ldots x_{0 n-1}
$$

wobei der erste Index sich jedesmal auf den Moment des Planens bezieht. Dieser Plan wird jedoch nicht ganz realisiert. Nur diejenigen Größen, deren Wert im Zeitabschnitt 0 fixiert werden muß, werden verwirklicht. Das sind natürlich erstens alle Angebots- und Nachfragemengen für den Zeitabschnitt 0; und zweitens alle späteren Mengen, welche natürlichen, technischen oder wirtschaftlichen Bindungen zufolge schon im Abschnitt 0 fixiert werden müssen. Die genannten „Pläne" sind aber noch abhängig von den Preisen und Preiserwartungen. Sie werden nun zu wirklichen, zahlenmäßigen Größen, wenn schließlich diese Preise einmal feststehen, was durch das Ausbalancieren von Nachfrage und Angebot geschieht. Mathematisch heiBt das, daB $p_{0}$ und damit die verschiedenen $x_{01}$ bestimmt werden durch die Gleichung

$$
X_{00}\left(p_{0}\right)=0
$$

wobei $X_{00}$ die Summe aller individuellen $x_{00}$ (mit positivem Vorzeichen im Falle der Angebotsmengen, mit negativem im Falle der Nachfragenmengen) ist; diese Gleichung besagt ja, daß $p_{0}$ so sein muß, daß Angebot = Nachfrage.

Wenn in der Weise ein Teil des „Wirtschaftsplanes $0^{\text {" }}$ realisiert ist, und der Anfang des zweiten Abschnittes gekommen ist, treten inzwischen vielleicht neue Umstände ein, haben sich auch vielleicht Ansichten, Erwartungen geändert, und so wird der ,Wirtschaftsplan $1 "$ :

$$
x_{11} x_{12} \ldots \ldots x_{1 n}
$$

sehr gut verschieden sein können vom ,Plan $0^{c} ; \mathrm{d}$. h. es braucht nicht $x_{01}=x_{11}$ zu sein. Auch von diesem Plan wird wieder ein bestimmter Teil realisiert usw. Die sukzessive realisierten Größen zusammen können wir den ,"wirklichen Wirtschaftsablauf" nennen.

Wir haben hiemit eine Arbeitshypothese dargelegt, die wohl von den meisten Forschern auch als die auf der Hand liegende Erweiterung des bekannten statischen Schemas gefühlt werden wird. Thre Brauchbarkeit muß sie aber natürlich erst bei der Lösung konkreterer Probleme beweisen, wenn möglich an Hand statistischer Daten. Nun ist die Fülle 
dynamischer Probleme selbstverständlich noch umfangreicher als die Zahl der statischen und fast zahllos. Eine Einteilung, sei sie noch so formell, ist also zum Utberblick der möglichen Richtungen erwünscht. Man kann für diesen Zweck eine Unterteilung in Probleme des dynamischen Angebots und in solche der dynamischen Nachfrage machen. Diese Trennung ist nicht gar so oberflächlich, wie sie wohl scheint. Hier wollen wir dann wählen und für dieses Mal nur ein Beispiel einer dynamischen Angebotsbildung behandeln. Ich hoffe später auf dynamische Nachfrageprobleme insbesondere noch zurückzulkommen.

\section{Der Zusammenhang zwischen Versorgungs- und Verbrauchsschwankungen von landwirtschaftlichen Produkten}

6. Als Anwendung der obigen Ausführungen auf mehr konkrete Probleme, möchte ich in diesem Abschnitt den Zusammenhang zwischen Versorgungsschwankungen und Verbrauchsschwankungen von Ackerbauprodukten näher betrachten. (Unter der Versorgung versteht man bekanntlich die Summe von Erntemenge und Vorrat aus alter Ernte.) Es besteht nämlich, wie z. B. in Abbildung 2 ersichtlich ist, ein Zusammenhang zwischen diesen beiden Größen, in dem Sinne, da $B$ sie einander nicht ohne weiteres gleich sind, sondern nur proportional schwanken. In einer streng komparativ-statischen Wirtschaft würde das ausgeschlossen sein; ein Vorrat würde nicht übertragen werden. Hier kann also wirklich ein dynamisches Problem vorliegen. Der Proportionalitätsfaktor ist weiter aus verschiedenen Gründen interessant. Die Abweichung zwischen Verbrauchs- und Versorgungsschwankungen, die er mißt, muß in der Handelssphäre (nicht in der Verbrauchssphäre), irgendwie verursacht werden, und also nicht, wie die Elastizität der Nachfrage letzterer Konsumenten, rein

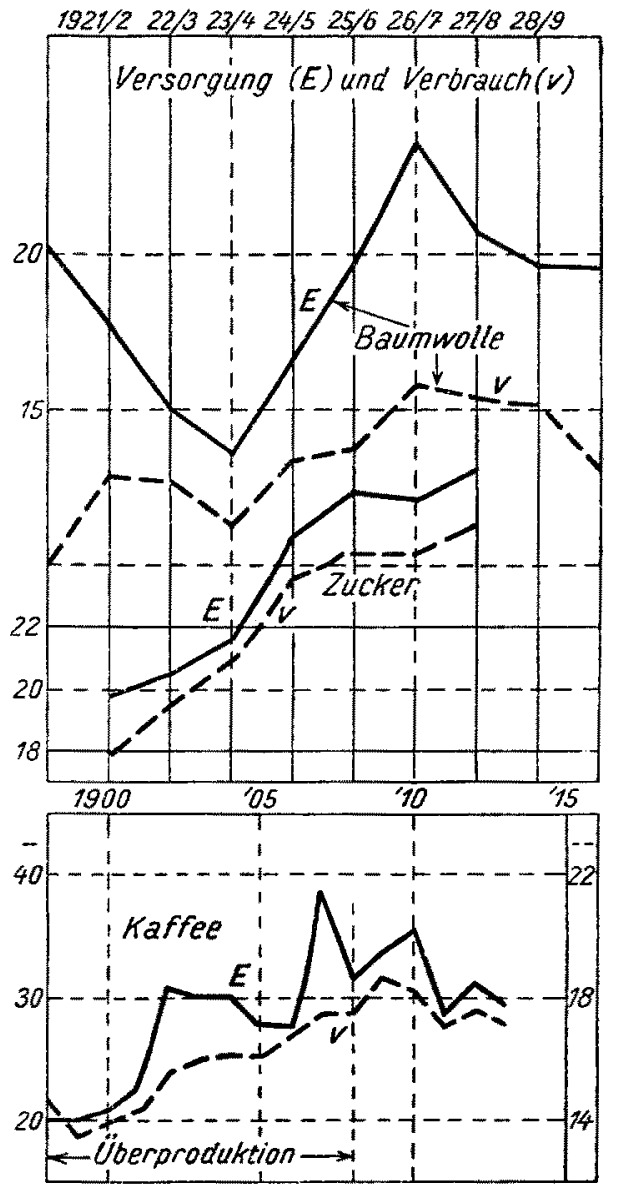

Abb. 2. Quellen: Baumwolle: International Cotton Bulletin; London und Cambriage Fco. nomic Service. Zucker: Int. Lendwirtsehaftsinstitut; London and Cambridgo Ficonomic Service. Kaffeo: Roth, a. a. 0 . 
psychisch bedingt sein, sondern mit der Struktur der Gewinnfunktionen des Handels zusa,mmenhängen und daher weiterer ökonomischer Analyse zugänglich sein. Die im folgenden zu gebenden „Abbildungen“ dieses Problems, die notwendigerweise stark vereinfachter Natur sein müssen, wollen jedoch nicht eine Lösung sein, sondern nur einen Weg zur Lösung angeben. Die wirkliche Lösung kann auch wahrscheinlich besser für jede einzelne Ware angestrebt werden und kann nur von Forschern gegeben werden, die bezüglich dieser Waren große technische und kommerzielle Kenntnisse haben. Es werden im folgenden also nur Lösungen für mehr oder weniger vereinfachte Fälle gegeben werden; und zwar wird mit einer außerordentlich vereinfachten Abbildung begonnen werden, welche dann in verschiedener Weise der Wirklichkeit mehr angepaßt werden wird.

7. Diese sehr vereinfachte Problemstellung habe folgende Charakteristika:

(1) Es sind nur Produzenten und Konsumenten, also keine Händler da, und es besteht nur ein Markt, wo Produzenten und Konsumenten einander treffen;

(2) Die Nachfrage der Konsumenten ist statischer Natur;

(3) Sie ist nur vom Preis im selben Augenblick abhängig und nicht z. B. explizite von der Zeit;

(4) Das Angebot ist dagegen dynamisch, und zwar in dieser Weise: die Anbieter (Produzenten) streben nach dem größtmöglichen Gewinn, über zwei Jahre berechnet $(\tau=2)$; während eines ganzen Jahres herrscht ein und derselbe Preis $\left.p_{t}{ }^{1}\right)$;

(5) Es besteht freie Konkurrenz auf beiden Seiten des Marktes;

(6) Die Anbaufläche (d. h. der Produktionsplan und damit die Produktionserwartung für spätere Jahre) ist konstant;

(7) Die Kosten der Vorrathaltung sind zu vernachlässigen.

Es wird weiter noch angenommen - vergleiche damit das im II. Abschnitt Gesagte - daß möglichst gute und schnelle Information besteht, neben absolut richtiger Einsicht aller Anbieter, wodurch erreicht wird, daß alle ,Erwartungen“", vernünftig" und für verschiedene Subjekte die gleichen sind.

Das Problem lautet: wenn die Funktion $f(p)$, die die Nachfrage in Abhängigkeit des Preises darstellt, und ferner die Ernte jedes Jahres nebst dem Anfangsvorrat im ersten von uns zu betrachtenden Jahre gegeben ist, wie groß ist dann der Verbrauch in jedem Jahr?

8. Die Lösung ergibt sich, wie man leicht einsieht, in folgender Weise. Im Anfang eines beliebigen Jahres $t$ mit einer Erntemenge von $e_{t}$ und einem ,carry-over" $u_{t}$ steht den Produzenten eine "Versorgung" $E_{t}=e_{t}+u_{t}$ zur Verfügung.

Da sie, gemäß (4), ihre Dispositionen immer für zwei Jahre machen, überlegen sie weiter, $\mathrm{da} B$ im nächsten $\mathrm{Jahr}(t+1)$ eine Erntemenge $e_{i+1}$

1) In der Sprache des II. Abschnittes dieses Aufsatzes ist also die Lānge eines Zeitabschnittes gleich einem Jahre angenommen und hat weiter die Ophelimitätsfunktion die Spezialgestalt des Gewinnes angenommen. 
zu erwarten ist, welche wir gemäß (6) gleich der mittleren Erntemenge über verschiedene Jahre $\bar{e}$ setzen können; für die zwei Jahre $t$ und $t+1$ ist also mit einem Gesamtangebot

$$
E_{t}+\bar{e}
$$

zu rechnen. Die Verteilung dieses Gesamtangebots über die zwei Jahre hängt von den relativen Preisen ab, die in diesen Jahren erwartet werden; nach (7) und unseren allgemeinen Annahmen wird das Angebot auf dasjenige der beiden Jahre gerichtet werden, in dem der höchste dieser beiden Preise erreicht wird. Das hat zur Folge, daß zwei Möglichkeiten auftreten. Erstens kann, wenn die Versorgung in gewissen - später zu präzisierenden - Hinsichten eine beschränkte ist, und daher $p_{t}$ höher angenommen wird als $p_{t+1}$, das gesamte im Anfang des Jahres $t$ zur Verfügung stehende Angebot $E_{t}$ auch wirklich angeboten werden. Der Verbrauch wird dann gleich der Versorgung sein, und der Preis $p_{t}$ so beschaffen sein, daß bei ihm Nachfrage gleich Versorgung ist, also

$$
E_{t}=f\left(p_{t}\right)
$$

Zweitens kann die Erwartung $\mathfrak{p}_{t}$ gleich sein dem $\mathfrak{p}_{t+1}$. Gemäß unserer Annahme der Vernünftigkeit der Erwartungen wird dann das Angebot so beschaffen sein, daß die Hälfte der Menge

$$
E_{t}+\bar{e}
$$

angeboten wird; gleiche Preise $p_{t}$ und $p_{t+1}$ sind ja nur dann vernünftig, wenn der Verbrauch für die beiden Jahre als gleich angenommen wird. Es wird in diesem Falle also der Verbrauch

$$
v_{t}=\frac{1}{2}\left(E_{t}+\bar{e}\right)=\bar{e}+\frac{1}{2}\left(E_{t}-\bar{e}\right)
$$

d. h. er beträgt um die Hälfte der Differenz zwischen der Versorgung und der normalen Ernte mehr als diese letztere: die ,Ưber versorg ung“" $E_{t}-\bar{e}$ wird nur zur Hälfte verbraucht. Der Preis folgt aus: $v_{t}=f\left(p_{t}\right)$. Einen dritten Fall gibt es nicht, weil das zurückhalten des gesamten Angebots bei einer relativ niedrigen Preiserwartung für das laufende Jahr den Preis gleich steigern würde und die Erwartung als unhaltbar zeigen würde. Man vergleiche hiezu die Abbildung 1, wo die Terminpreise wirklich fast immer entweder gleich den augenblicklichen Preisen oder niedriger als diese sind.

Die Lösung im zweiten Fall lehrt uns zugleich, wo die Grenze für sein Zutreffen liegen muß. Das wird der Fall sein, wenn $E_{t}$ unter $\vec{e}$ liegt; in diesen Umständen trifft der erstens genannte Sachverhalt ein. Das geschieht also nur dann, wenn die Versorgung - d. h. Ernte plus alter Vorrat - unter der mittleren Ernte $\bar{e}$ liegt; dazu muß die Ernte dann a fortiori unter $\bar{e}$ liegen, was bei Gau Bischer Verteilung der Erntemengen um $\bar{e}$ herum, also in weniger als in der Hälfte der Fälle zutreffen würde. 
Allgemein zusammengefaßt ist die Lösung deshalb: die Verbrauchsschwankungen sind kleiner als die Versorgungsschwankungen, der Proportionalitätsfaktor liegt zwischen $1 / 2$ und $1^{1}$ ).

Es ist deutlich, daß zwischen Ernte- und Verbrauchsschwankungen im vorliegenden Fall kein eindeutiger Zusammenhang besteht: dieser Verband ist stochastisch. Eine gewisse Idee hierüber bildet man sich jedoch durch Betrachtung des Verhältmisses zwischen den mittleren Abweichungen der Erntemengen bzw. der Verbrauchsmengen. Nähme man an, daß der Verband zwischen Verbrauch und Versorgung nur durch (B) gegeben würde, daB also die Fälle (A) ausgeschlossen seien ${ }^{2}$ ), so fände man für das obengenannte Verhältnis $\sqrt{3}$, also weniger als das Verhältnis der mittleren Abweichungen der Versorgungs- und der Verbrauchsmengen $^{3}$ ).

Wie gesagt, wollen wir jetzt den einfachen Fall, den wir bis jetzt betrachtet haben, nach verschiedener Seite hin verallgemeinern. Und zwar wollen wir, der Reihe nach, die sieben obengenannten Einschränkungen durch allgemeinere Annahmen ersetzen.

9. (1) Zwischen Produzenten und Konsumenten befinden sich irgendwelche „Händler", welche die Ware von den Produzenten kaufen und den Konsumenten verkaufen. Eine einigermaßen erschöpfende Behandlung dieses Falles müssen wir leider auf später verschieben. Es wäre hier nämlich angemessen, von den „Händlern" anzunehmen, ihre Nachfrage sei nicht statisch, sondern dynamisch. Das würde der Wirklichkeit gewiß am besten entsprechen. Nur unter sehr vereinfachenden Annahmen läßt sich jedoch die Behandlung dieses letzten Problems hier durchführen. Man stöBt in den anderen Fällen nicht nur auf mathematische Schwierigkeiten, welche ziemlich gro $B$ sind, sondern auch die Fülle der Möglichkeiten ist hier so groß, daß es wenig $Z$ weck hätte, darüber zu

1) Wie man sieht, ist der Zusammenhang, welcher hier zwischen $v$ und $E$ besteht, ein schönes Beispiel dafür, wie eine gebrochene Linie als Funktionsabbildung einen einfachen Sinn haben kann.

2) Man kann sich das wohl denken, wenn irgendein Reserverorrat bestände, welcher in den Berechnungen der Anbieter nicht eingänge.

3) Der Beweis geschieht mit Hilfe der Annahme, daß die Erntemenge unabhängig ist vom am Anfang des Jahres anwesenden Utberschuß.

Es ist nämlich:

also $2\left(u_{t}-\bar{u}\right)=e_{t-1}-\bar{e}+u_{t-1}-\bar{u}$

$$
u_{t}=\frac{e_{t-1}+u_{t-1}-\bar{e}}{2}
$$

$4\left(u_{t}-\bar{u}\right)^{2}=\left(e_{t-1}-\bar{e}\right)^{2}+\left(u_{t}-1-u\right)^{2}+2\left(e_{t-1}-\bar{e}\right)\left(u_{t-1}-\bar{u}\right)$

Wenn wir weiter bedenken, daß $\sigma_{u}^{2}=\overline{\left(u_{t}-\bar{u}\right)^{2}}$, und unserer Annahme gemäB $\overline{\left(e_{t}-1-\bar{e}\right)\left(u_{t}-1-\bar{u}\right)}=0$, so folgt:

$$
4 \sigma_{u}^{2}=\sigma_{e}^{2}+\sigma_{u}^{2} \text { oder } \sigma_{e}^{2}=3 \sigma_{u^{2}}^{2}
$$

In derselben Weise beweist man aus der Beziehnng $2 v_{t}=e_{t}+u_{t}+\bar{e}$ : woraus schließlich:

$$
4 \sigma_{v}^{2}=\sigma_{e}^{2}+\sigma_{u}^{2}
$$

$$
\sigma_{v}^{2}=\frac{1}{3} \sigma_{e}^{2}
$$


entscheiden, bevor statistisches Material bestimmter Fälle dazu nötigt. Teilweise rächt sich hier wohl der Umstand, daß auch in der einfachen statischen Theorie die Theorie des Handels sehr vernachlässigt worden ist. Die Möglichkeiten, die hier liegen, nämlich diese statische Theorie durch Spezialwahl der Ophelimitätsfunktion (welche dann gleich dem Gewinn des Händlers, also einem durchsichtigen mathematischen Ausdruck zu nehmen wäre), zu genaueren Aussagen über die hier liegenden Probleme zu bringen, sind noch selten benutzt worden und warten größtenteils noch auf Bearbeitung. Weil aber in diesem Aufsatz andere Momente im Vordergrund stehen sollen, so seien hier nur die einfachsten Fälle berührt.

$\mathrm{Zu}$ diesen gehört wohl erstens der Zustand, in dem die Produzenten irgendwie gezwungen sind, die Ernte sofort den Händlern zu verkaufen. Fs übernehmen dann einfach die Händler die Rolle der Produzenten im obigen Schema, und die Lösung bleibt dann unverändert.

Zweitens kann man noch mühelos folgende Möglichkeit betrachten. Die Nachfrage der Händler sei nämlich, ebenso wie diejenige der Konsumenten, nur vom gleichzeitig herrschenden Preis abhängig. Sie muß der genauen Form nach gar nicht mit der Konsumentennachfrage identisch sein, kann z. B. viel elastischer sein als diese (unter Umständen kann auch eine dynamische Nachfrage ungefähr diese Gestalt besitzen). Aus dem oben ausgeführten läßt sich dann leicht ersehen, daß angenommen, daß die Händler auch dynamisch (mit einem $\tau=2$ ) verkaufen, der Verbrauch der Konsumenten in derselben Beziehung steht zum ,Verbrauch“ der Händler (womit einfach deren Käufe gemeint sind), wie dieser letztere zur Erntebewegung. Die Abschwächung wird jetzt also quadriert; d. h. z. B., daß nun die Verbrauchsschwankungen ein Drittel (statt $\frac{1}{\sqrt{3}}$ ) der Ernteschwankungen wären, wenn man von den Fällen der Formel (A) absehen könnte.

10. (2) Die Nachfrage der Konsumenten ist nicht statischer Natur.

Die wichtigste Änderung, welche hiedurch in unseren Ưberlegungen erfolgt, ist wohl, daß jetzt die Nachfrage nicht nur vom gleichzeitig herrschenden Preis, sondern auch vom Preisverlauf irgendwie abhängig sein muß. Teilweise aus denselben Gründen als den unter (1) genannten, muß von einer einigermaßen erschöpfenden Behandlung des so entstehenden Problems abgesehen werden: die Nachfragefunktion, welche im allgemeinen hier zu benutzen wäre, ist sehr verwickelt. Wir wollen daher wieder nur einen einfachen Spezialfall betrachten, nämlich den, bei welchem die Nachfrage außer vom Augenblickspreis, auch vom Preis des Vorjahres bestimmt wird. Hiemit schließen wir bei den von Amoroso ${ }^{1}$ ) und $\operatorname{Roos}^{1}$ ) gebrauchten dynamischen Nachfragefunktionen an,

1) Amoroso: Intorno alle determinazione empirica delle leggi della domanda e dell' offerta, Giomale degli Economisti, November 1930, S. 941, und frühere Arbeiten; Roos: A Mathematical Theory of Competition, Am. Journal of Math. XLVII (1925), S. 163; Evans: A Simple Theory of Competition, Am. Math. Monthly, XXIX (1922). 
wo neben $p$ die erste Ableitung von $p$ nach der Zeit als unabhängige Variable genommen worden ist.

Auch hier wollen wir dann noch den Spezialfall nehmen, bei dem die Nachfrage in den beiden $p$ linear ist, und zwar:

$$
v_{t}=a-b p_{t}+c\left(p_{t}-p_{t-1}\right)
$$

Dadurch, daß man $c=0$ nimmt, verschwindet das Glied mit der Preisdifferenz - das speziell dynamische Glied - und es bleibt eine Nachfragefunktion, welche statisch sein kann.

Eine leichte Rechnung zeigt, daß bei Annahme von $p_{t}=p_{i+1}$ und bei Annahme "vernünftiger" Erwartungen statt der Formel (B) jetzt gefunden wird:

$$
v_{t}=\alpha\left(a-b p_{t-1}\right)+(1-\alpha) \frac{E_{t}+\bar{e}}{2}
$$

wobei

$$
\alpha=\frac{c}{2 b-c}
$$

Man sieht leicht ein, daß für $c=0$, also bei Ausfallen des speziell dynamischen Gliedes in der Nachfrage, das alte Resultat wieder vorliegt. Dagegen wird dieses ,um so stärker ersetzt" durch $a-b p_{t-1}$, je mehr $c$ wächat. Die Bedeutung von $a-b p_{t-1}$ ist offenbar: die ,statische" Nachfrage, welche für einen Preis gleich dem des Vorjahres herrschen würde. Für $\alpha=1$, also $b=c$, bekommt man einfach eine verzögerte "statische" Nachfrage. Wo auch das erste Glied der obigen Formel (B1) von Jahr auf Jahr schwankt, ist es nicht leicht, gleich einzusehen, ob die Schwankungen des Verbrauchs in diesem dynamischen Fall größer oder kleiner sind, als im einfachen, statischen Fall. Fine nähere Rechnung lehrt, daß das Verhältnis der mittleren Abweichungen von Verbrauch und Versorgung - das im einfachen Fall $\frac{1}{2}$ war - je nach dem. Wert von $\alpha$ kleiner oder größer als $\frac{1}{2}$ werden kann.

11. (3) Die Nachfrage hängt neben dem Preis auch explizite von der Zeit ab.

Nehmen wir zuerst an, daß die Nachfrage jedes Jahr um einen konstanten Wert zunimmt, wenn sich der Preis nicht ändern würde (wenn dieser sich wohl ändert, kommt eine entsprechende Änderung dazu). Das heißt also, die Nachfrage habe einen geraden Trend; mathematisch :

$$
v_{t}=f\left(p_{t}\right)+\alpha t .
$$

Man rechnet leicht nach, daß, vorausgesetzt daß dieser Trend allen Betroffenen bekannt ist, am Resultat der Formel (B) nichts geändert wird, wenn man $\bar{e}$ jetzt als den Trendwert auffaßt.

Fine zweite Mögliohkeit wäre, daß die Nachfrage jedes Jahr um einen konstanten Prozentsatz zunähme. Das Resultat ändert sich dann leicht. 
Schreibt man

so kommt statt (B):

$$
v_{t}=\alpha^{t} f\left(p_{t}\right)
$$

$$
v_{i}=\frac{E-\bar{e}}{1+\alpha}+e
$$

wobei $\bar{e}$ wieder den Trendwert der Ernte bezeichnet.

Der Proportionalitätsfaktor hat sich also von $\frac{1}{2}$ auf $\frac{1}{1+\alpha}$ geändert; wobei $\alpha-1$ in den meisten praktischen Fällen aber sehr klein gegenüber 1 sein wird, daher ist der Unterschied meistens belanglos zu achten.

Wir haben angenommen, daB die Abhängigkeit der Nachfrage von der Zeit eine einfache und allen Subjekten (deshalb) bekannte sei. Es ist nämlich deutlich, daß eine verwickelte, schnell wechselnde Abhängigkeit, welche (dann) nicht den Subjekten bekannt ist, einer zufälligen Bewegung sehr nahe kommt und unter Umständen identisch ist mit der rein zufälligen Bewegung, welche wir schon bei den Erntemengen angenommen haben und welche deshalb nichts Neues in das Problem bringen würde. Die Bedeutung der angenommenen Abhängigkeit der Nachfrage von der Zeit ist auch noch diese, daß sie diejenigen Veränderungen der Marktlage darstellt, welche richtig vorausgesehen werden (können), während demgegenüber die Erntebewegung den nicht voraussehbaren Teil der Marktlage darstellen kann. Je nach der relativen Größe dieser beiden "Teile" hat man Fälle mit „besserer" oder „,schlechterer" Voraussichts-Möglichkeit und also mit relativ kleineren oder größeren prozentuellen Fehlern in der Abschätzung. Je kleiner die jährlichen Ernteschwankungen gegenüber dem Trend sind, um so mehr bilden die Erntemengen eine, ,gebundene" (G-)Reihe im Sinne Andersons, je größer diese Schwankungen gegenüber dem Trend sind, um so mehr bilden die Erntemengen eine ,zackige" (Z-)Reihe').

12. (4) Das Gesichtsfeld der Anbieter ist größer oder kleiner als zwei Jahre.

Es ist leicht einzusehen, daß statt der Formel (B), wenn $\tau$ willkürlich gedacht wird, zu lesen ist:

$$
v_{t}=\bar{e}+\frac{1}{\tau}\left(E_{t}-\bar{e}\right)
$$

d. h. daß von der Utberversorgung jetzt ein um so kleinerer Teil verbraucht wird, je nachdem $\tau$ größer ist. Das interessante an diesem Ergebnis - das natürlich dem aufmerksamen Leser bei der Behandlung unseres einfachen Schemas eingeleuchtet haben dürfte - ist wohl, daß der „Dämpfungsfaktor" zunächst also nicht von der Elastizität der Nachfrage oder irgendwas dergleichen abhängt; sondern vom „Gesichtsfeld" der Produzenten. Erst in den Preisschwankungen wird die Elastizität der Nachfrage bemerkbar.

1) Anderson: Die Korrelationsrechnnng in der Konjunkturforschung, Veröffentlichungen der Frankfurter Gesellschaft für Konjunkturforschung, Heft 4, 1929. 
Man rechnet weiter leicht nach, daß für den Fall, daß für jedes Jahr der Zusammenhang von Verbrauch und Versorgung durch (B) gegeben wäre (man erinnert sich, daß das in „,knappen“ Jahren nicht der Fall ist), das Verhältnis der mittleren Abweichungen von bzw. Verbrauch und Ernte bestimmt ist durch:

$$
\sigma_{v} \sqrt{2 \tau-1}=\sigma_{e}
$$

Dieses Verhältnis nimmt also bei zunehmendem $\tau$ langsamer ab, als das Verhältnis des jeweiligen ,Ưberverbrauchs" zur ,Ubberversorgung ' ${ }^{\text {}}$ ).

13. (5) Es herrscht keine freie Konkurrenz am betrachteten Markte.

Praktisch ist wohl meistens die Möglichkeit monopolistischer oder halbmonopolistischer Produktion wichtig:-weniger die Möglichkeit monopolistischer Konsumtion.

Beginnen wir mit der Annahme, daß die Produzenten in einem monopolistischen Verband organisiert sind. Man überzeugt sich mühelos davon, daß dieses an unserem einfachen Schema nichts ändert: auch jetzt ist entweder $v_{t}=E_{t}$ oder $v_{t}=\frac{E_{t}+\bar{e}}{2}$. Dasselbe gilt, mutatis mutandis, für den Fall, wo $\tau$ willkürlich ist.

Dagegen erscheinen kleine Abweichungen zwischen dem monopolistischen und dem Konkurrenzfall, sobald die komplizierten Annahmen gemacht werden, wovon oben unter (2) oder (3) genannt wurden. In diesen Beispielen fällt die Gleichheit der Preiserwartungen weg: es ist vorteilhafter für den Monopolisten, in den beiden Wirtschaftsjahren, die er immer zugleich betrachtet, um verschiedene Preise zu fragen. Damit werden dann auch die Resultate leicht abgeändert, obgleich in der Hauptsache wenig Unterschiede auftreten. Natürlich bewegen sich aber die Verbrauchs- und Ernteschwankungen um ein anderes Niveau, das statisch-monopolistische. Man wird sich überzeugen können, daß auch danach in den unter (6) und (7) zu nennenden verallgemeinerten Problemen ein leichter Unterschied zwischen Konkurrenz und Monopol zu konstatieren ist.

Qualitativ dasselbe gilt auch für die weitere Annahme, die man bezüglich der Marktorganisation zum Ausgangspunkt wählen kann, nämlich die der beschränkten Konkurrenz.

1) Es scheint (siehe Abb. 2, untere Kurve), daß bei Kaffee in den Jahren 1898 bis 1908 ein größeres $\tau$ als in den Jahren 1908 bis 1913 maßgebend gewesen ist. Es ist in dieser Hinsicht interessant, daß bis 1908 eine deutliche Utberproduktion im Kaffee geherrscht hat (vgl. Roth: Die Überproduktion in der Welthandelsware Kaffee 1790 bis 1929, Jena, 1929), während nach 1908 die Vorräte wieder leicht vermindert sind. Man kann sich denken, daB in Jahren der Utberproduktion die Händler gezwungen sind, auf längere Sicht zu wirtschaften, als in Jahren des Gleichgewichts. Diese Bemerkung soll aber nicht mehr als eine Anregung sein. 
14. (6) Der Produktionsplan für das nächste Jahr ist abhängig vom Preis des laufenden Jahres.

Obgleich die in unserem einfachen Schema gemachte Hypothese, der Produktionsplan (die Anbaufläche) und also die Produktionserwartung sei konstant, in vielen praktischen Fällen nahezu verwirklicht ist, so sind doch auch die Fälle zahlreich, wo die Anbaufläche in Abhängigkeit vom Preisstand verändert wird ${ }^{1}$ ). Es hat also einen guten Sinn auch nach dieser Seite hin, eine kleine Verallgemeinerung zu versuchen. Dabei nehmen wir an, daß die Produzenten ihre Anbauflächen so wählen, daß die Ernteerwartung (d. h. die mittlere Ernte auf dieser Fläche) ${ }^{2}$ ) statt $\bar{e}$ sein wird

$$
e_{t+1}=\bar{e}+\varepsilon\left(p_{t}-\bar{p}\right)
$$

wobei $\varepsilon$ eine positive Konstante und $\bar{p}$ der mittlere Preis ist. Man rechnet wieder ohne weiteres nach, $\mathrm{da} B$ an Stelle von (B) jetzt zu schreiben ist:

$$
v_{t}=\bar{e}+\frac{b}{2 b+\varepsilon}\left(E_{t}-\bar{e}\right)
$$

woraus ersichtlich ist, daß bei wachsendem $\varepsilon$ die Verbrauchsschwankungen abnehmen.

In der wirtschaftlichen Praxis kommt in der Gleichung (C) oft ein Glied mit $p_{t-1}$ dazu; dieses beeinflußt aber das Schwankungsverhältnis von $v$ und $E$ nicht.

Wir wollen noch darauf hinweisen, daB die Stabilisierung des Verbrauchs, welche gemäß Formel (D) möglich sein soll - abbesehen von "Mißerntejahren", wo $E<\bar{e}-$, nur dann der Wirklichkeit entspricht, wenn in der Tat, wie angenommen wurde, der Koeffizient $\varepsilon$ allen Anbietern bekannt ist $^{3}$ ).

15. (7) Die Kosten der Vorrathaltung werden berücksichtigt.

Nehmen wir an, daß die Kosten pro Jahr und pro Einheit für das Aufbewahren des betrachteten Produkts auf $r$ kommen, so ist deutlich, daß ein Anbieter jetzt erst dann seine Ware bis ins nächste Jahr zurückhalten wird, wenn seine Preiserwartung für dieses nächste Jahr um r oder mehr den Gegenwartspreis übertrifft:

$$
p_{t+1}>p_{t}+r
$$

Dieses hat zur Folge, daß die Ausgleichung des Verbrauchs einer gewissen Reibung unterzogen wird, und also die Schwankungen des Verbrauchs größer werden, als im einfachen Fall. Es wird von den

1) Vgl. vor allem Bean, L.: The farmers reponse to price, Journal of Farm Economics, 1929, S. 368.

2) Wir nehmen also an, daß die mittlere Ernte die wahrscheinlichste ist.

3) Wenn aber der Zustand so weit gekommen ist, wird man vielleicht $\varepsilon$ nach andern Gesichtspunkten feststellen. Auf die hieher gehörigen planwirtschaftlichen U̇berlegungen gehen wir aber hier nicht ein. 
Größenordnungen abhängen, in wie weitem Ausma $B$ dies der Fall ist. Es seien daher einige Zahlen genannt. Ezekiel nennt in seiner Arbeit „,A Statistical Examination of the Problem of Handling Annual Surpluses of Non-perishable Farm Products" eine Zahl von 9 cents für Vorratkosten (gegenüber einem Preis von rund 80 cents) für Weizen, welche Zahl nach ihm ziemlich hoch angenommen worden 1st. In einer holländischen Zeitschrift werden die Vorratkosten für ein Jahr für Kartoffeln auf etwa $5 \%$ des Preises angenommen.

Es soll noch daran erinnert werden, daB im Gegensatz zur Konkurrenz beim Monopol schon bei einem Unterschied von $\frac{1}{2} r$ das Zurückhalten des Angebots vorteilhaft wird ${ }^{1}$ ). Man kann dies leicht nachrechnen; die Ursache dieses vielleicht paradoxen Resultates ist darin gelegen, daß der Monopolist mit seiner Angebotsmenge zugleich auch den Preis beeinflußt, und das Zurückhalten einer kleinen, zusätzlichen Menge also nicht nur direkt, sondern auch indirekt, über den Preis, den Gewinn abändert.

16. Ich will die Behandlung dieses Beispiels und damit diesen Aufsatz mit einigen kurzen Bemerkungen ohne Zusammenhang schließen.

Erstens kann bemerkt werden, daß nicht nur durch „Kurzsichtigkeit“ der Anbieter der Fall zu einem statischen werden kann, wobei einfach die ganze Finte jedes Jahr verbraucht wird, sondern auch dadurch, $\mathrm{daB}$ die Ware nicht länger als ein Jahr aufbewahrt werden kann (wie z. B. Gemüsearten, in gewisser Hinsicht auch Kartoffeln).

Zweitens soll nochmals daran erinnert werden, wie sehr die obige Abbildung, trotz einiger Verallgemeinerungen, eine vereinfachte bleibt. So ist vom Preisablauf innerhalb des Jahres völlig abgesehen worden; von Fällen, wo $\tau$ für verschiedene Tauschende verschieden ist, ebenso; von der perspektivischen Verkleinerung ist nur ein grobes Bild gegeben worden usw. Trotzdem scheint es, daB gewisse speziell dynamische Züge richtig zum Ausdruck gekommen sind und vielleicht sogar besser durch die Vermeidung aller Komplikation. Die wirkliche Klärung des Preisablaufs einzelner Waren kann jedoch nur durch Spezialforschung gefunden werden; hoffentlich wird der dargelegte Mechanismus hiezu auch einmal beitragen können.

1) Wenigstens wenn entweder $r$ klein ist gegen $p$ oder die Nachfragefunktion eine lineare. 